\title{
HOW VALID ARE THE BIOLOGICAL AND ECOLOGICAL PRINCIPLES UNDERPINNING GLOBAL CHANGE SCIENCE?
}

\author{
Anastassia Makarieva1, Victor G. Gorshkov', \\ Brendan Mackey ${ }^{2}$ and Vadim V. Gorshkov ${ }^{3}$ \\ ${ }^{1}$ Petersburg Nuclear Physics Institute, 188300, Gatchina, St-Petersburg, Russia, \\ fax+78127131963,e-mail: elba@infopro.spb.su \\ ${ }^{2}$ Australian National University, Department of Geography, Faculty of Science, $S$ \\ chool of Resource Management and Environmental Science, Canberra ACT 0200 Australia, \\ fax+61 62493770,e-mail: Brendan.Mackey@anu.edu.au \\ ${ }^{3}$ Komarov Botanical Institute, 2 Prof. Popov Str., 197367, St-Petersburg, Russia, \\ fax+7812 2344512,e-mail: Dima@IB6521.spb.edu \\ Corresponding author: Dr. Anastassia Makarieva, e-mail: elba@infopro.spb.su, \\ tel. +78125502279 ,fax: +78127131963 .
}

\begin{abstract}
The prevailing scientific approach to investigating and understanding the environmental consequences of human-induced global change is underpinned by two basic biological principles. First, the principle that species genetically adapt to changing environment conditions. Second, the principle that nutrients present in the environment in the smallest relative concentrations limit biological productivity.

We contend that both principles have been formulated based on the results of investigations into either artificially selected organisms, or anthropogenically perturbed landscapes. In both these cases, organisms are studied outside their natural ecological niche. We argue that natural ecosystems do not conform to the above two principles. Non-perturbed biota of natural ecological communities form and maintain optimal environment conditions by buffering the flux of primary environmental resources that would otherwise randomly fluctuate as the result of purely physical processes. In such a biotically-mediated environment the availability of nutrients does not limit biological productivity. Critically, the capacity of the biota to regulate local environment conditions obviates the need for species to continually adapt to random environmental fluctuations.

We illustrate how the failure to distinguish between the functioning of perturbed and unperturbed biota prevents the development of policies and strategies that will lead to the long term resolution of the global ecological crisis.
\end{abstract}

Key words: biotic regulation of the environment, unperturbedecosystems, biodiversity, climate stability, global change, limiting principle, adaptation 


\section{INTRODUCTION}

The accelerating anthropogenic transformation of the global environment is causing growing concern amongst both scientists and the general public. Politicians and policy makers burdened with the responsibility for decisions that ultimately may affect billions of human lives are beginning to respond as witnessed by recent international commitments such as The Biodiversity Convention (Glowka et al. 1994) and the Kyoto Protocol (United Nations 1998). Consequently, scientific estimates and forecasts have begun to play a global political role. This imposes a huge responsibility on all scientists, and life scientists in particular given the vital role of biological systems in the maintenance of global life support systems (Walker \& Steffen 1997).

Most resources allocated today to biological disciplines within the framework of global change science are spent on the generation and improvement of empirical data bases and mathematical models that can be used to simulate global biophysical processes and systems (e.g. Alcamo 1994). However few, if any, efforts are aimed at verification of the conceptual principles that form the theoretical foundations of the modern biological and ecological sciences and their application to the science of global change. If these theoretical foundations are faulty, then resultant biases in global change research may become a source of dangerous errors. However advanced, no information system can be expected to generate reliable quantitative predictions, if the basic principles underlying system conceptualisation and data interpretation prove to be flawed.

In this article we argue for serious reconsideration of two basic principles that constitute foundational paradigms for contemporary life sciences, namely, (1) the limiting principle, and (2) the genetic adaptation principle. Re-evaluation of these paradigms in turn demands reconsideration of other generally accepted biological and ecological scientific concepts. A new perspective emerges which has profound implications for global change science, and ultimately for the kinds of policies and strategies needed to rectify global environmental problems.

\section{THE LIMITING PRINCIPLE}

The limiting principle refers to the generally accepted principle that biological functioning is nutrient limited. It may be defined as follows.

Functioning of the biota (biota - flora and fauna of natural ecosystmes) is dependent upon the availability of major nutrients (carbon, nitrogen, oxygen, phosphorus, iron etc.). All these elements enter biochemical reactions in non-random (stoichiometric) proportions. The least abundant nutrients limit the rate of biochemical reactions. Increase or decrease of concentrations of the limiting nutrients has a drastic effect upon functioning of the biota causing increasing or decreasing biological productivity. In contrast, any change in concentrations of elements that are present in the environment in greater amounts than those required by stoichiometric ratios with respect to the limiting nutrient, do not significantly influence functioning of the biota. In global change science practice, the task of investigating possible biotic response to environmental change is thus often restricted to determining the effects of limiting nutrients.

For example, if carbon is the limiting nutrient for the terrestrial biota, the latter may, in response to the human-induced change of atmospheric carbon concentration, increase production of organic matter thus withdrawing excessive carbon from the 
atmosphere and depositing it in the form of organic matter. If, on the other hand, carbon is not a limiting nutrient (i.e. it is already present in excess), the terrestrial biota will ignore changes in the atmospheric carbon content and can be excluded from global change models.

Laboratory experiments with plants in pots (IGBP 1997) show that green plants do increase the rate of photosynthesis in response to elevated $\mathrm{CO}_{2}$ concentrations. Although consistent empirical evidence confirming similar behaviour of natural ecosystems on a global scale is lacking, these experiments had led to inclusion of the terrestrial biota into the global budget of carbon changes.

Another critical global reservoir of organic carbon is the oceanic pool of dissolved organic carbon, which is approximately equal to the atmospheric carbon pool. However, in the ocean, inorganic carbon seems to be present in proportions far in the excess of those ratios needed for synthesis of organic matter by the marine biota (see, however, Degens et al. 1968). It has therefore been assumed that productivity of the marine biota is limited by phosphorus and other elements that are used by the biota in relatively small concentrations. On this basis, oceanic biota was considered to be severely limited in its ability to respond to the increase of atmospheric $\mathrm{CO}_{2}$ (which is accompanied by an increase in the dissolved inorganic carbon concentration in the surface waters). As a result, the oceanic biota has been traditionally excluded from models of the global carbon budget (Keeling et al. 1996; Heimann \& Maier-Reimer 1996).

In other words, the limiting principle prohibits consideration of any possible largescale reactions of the biota to elevated $\mathrm{CO}_{2}$ other than an increase or decrease in the total primary production. If one accepts that the oceanic biotic response may be more diverse and complicated than predicted by the limiting principle, it is possible to obtain quite a different picture of the modern global carbon cycle, see Fig. 1. For example, the stabilising reaction of the oceanic biota to the anthropogenic disturbance of the atmospheric composition may take the form of changed proportions in production of long-lived and short-lived biomatter, the overall productivity remaining unchanged. If more long-lived biomatter is produced, one may expect to find a significant organic carbon sink in the ocean. Its magnitude can deduced from the available data on atmospheric $\mathrm{O}_{2} / \mathrm{N}_{2}$ ratio change and the known different stoichiometric $\mathrm{C} / \mathrm{O}$ ratios in the biotas of land and ocean as well as in the fossil fuel.

As one can see from Fig. 1, such a consideration makes it possible to account for the modern global carbon budget without assuming the existence of a substantial carbon sink on land. Such a sink can hardly be assigned to the terrestrial biota. The latter is significantly transformed by humans, while it is well-known that the exploited lands add carbon to the atmosphere, mostly due to deforestation and soil erosion.

Given the extent to which the limiting principle influences global change science, issues associated with whether or not they are scientifically valid are of more than academic interest. The following questions appear therefore to be justified:

- how valid is the limiting principle for description of global biota?

- if we confine ourselves to the limiting principle, do we overlook important features in biota-environment interaction?

- is there a firm scientific basis to dismissing the possibility of an oceanic biotic sink? 


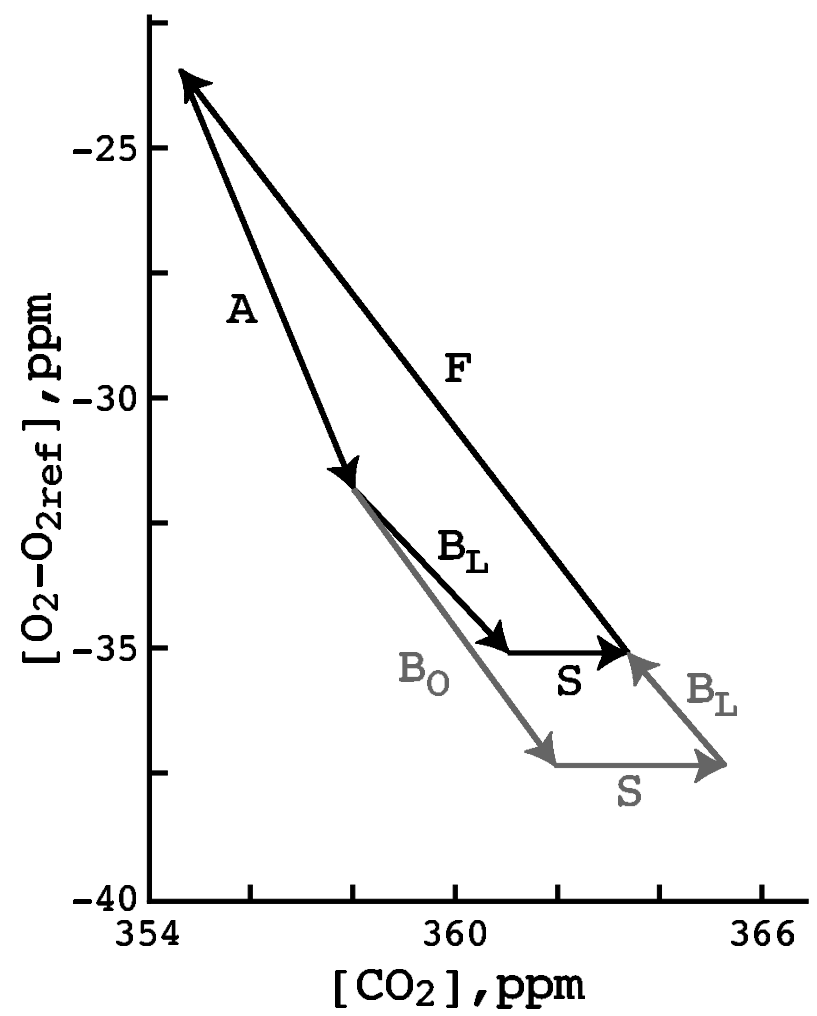

Figure 1. Possible different views on the global carbon cycle as dictated by acceptance/rejection of the limiting principle.

Vectors indicate the three-year (1991-1994) changes in carbon and oxygen content in the major global reservoirs: $\mathrm{A}$ - atmosphere, $\mathrm{F}$ - fossil fuel, $\mathrm{B}_{\mathrm{L}}$ - land biota, $\mathrm{S}$ - dissolved inorganic carbon of the ocean, $\mathrm{B}_{\mathrm{O}}$ - oceanic biota (dissolved organic carbon). Vector slopes are determined from the stoichiometric ratios $\alpha \equiv \mathrm{O}_{2} / \mathrm{CO}_{2}$ for the land biota $(\alpha=1.10 \pm 0.05)$, oceanic biota $(\alpha=1.30 \pm 0.03$, Redfield ratio) and

fossil fuel $(\alpha=1.38 \pm 0.04)$, and by direct measurements for the atmosphere

$$
(\alpha=2.2 \pm 0.2 \text {, Keeling et al. 1996). }
$$

Black vectors: Global carbon cycle as predicted by the limiting principle (from Keeling et al. 1996): the oceanic biota vector $\mathrm{B}_{\mathrm{O}}$ is missing, the land biota $\mathrm{B}_{\mathrm{L}}$ becomes a large net sink of carbon.

Grey vectors: Global carbon cycle if one accounts for possible reaction of the oceanic biota (from Gorshkov \& Makarieva 1998): the oceanic biota ensures a considerable sink of carbon, the land biota represents a net source of carbon to the atmosphere in accordance with direct measurements of carbon flows from cultivated lands. (The inorganic carbon sink $\mathrm{S}$ (green) was determined from ${ }^{13} \mathrm{C} /{ }^{12} \mathrm{C}$ data under the assumption that the rate of inorganic carbon uptake by the ocean grows proportionally to the relative increment of atmospheric $\mathrm{CO}_{2}$.) 


\section{IMPLICATIONS FOR GENETIC ADAPTATION TO CHANGING ENVIRONMENT}

By definition, if one of the nutrients is limiting, other nutrients are present in the environment in excess. This means that their consumption by the biota operates in a regime of saturation, where both the production and decomposition of organic matter become independent of changes in the environmental concentration of the nonlimiting nutrient. In such a case no biotic control of non-limiting nutrients is possible, because whatever changes their concentrations undergo, the biota does not react to them. As a result, concentrations of non-limiting nutrients drift randomly under the influence of external abiotic factors.

Thus, if the limiting principle is accepted, it follows that environmental conditions cannot be under biotic control. It follows that to persist, the natural biota of Earth must be capable of adapting to randomly changing physical fluxes and hence environmental conditions. This we may call the genetic adaptation principle which is one of the most broadly accepted principles in biological science today, and a major pillar supporting the current approaches to global change science.

The genetic basis of adaptation is supported by the observed genetic polymorphism (i.e., genetic non-identity of individuals in a population) and mutability (i.e., appearance of new genetic options, not found in parental lines). The best-adapted individuals are, by definition, those claiming the maximum of the available resources and producing the maximum number of offspring. Genetic variants imparting the highest reproductive capacity to their carriers, propagate in the population. Gradual accumulation of hereditary genetic changes finally leads to the fact that individuals of a given species acquire specific traits of a new species. The primary driving force of evolution is, consequently, a random change of the environment that brings about changes in the natural selection pressures (see, for example, Dobzhansky 1970).

In the meantime, independent development of both empirical and theoretical global change research has outlined the possibility of a different approach to the problem of biota-environment interactions, where the natural biota is largely responsible for formation and maintenance of a life-compatible environment on the planet. Functioning of natural ecological communities compensates all external environmental disturbances, stabilising the environment in a certain optimum state. (Information needed for such regulation should be then coded in the genomes of biological species that form the ecological community.) Such a way of functioning of natural ecosystems obviates the need for organisms to adapt to changing environments. It also follows that anthropogenic transformation of natural genetic programs of species in the course of artificial selection, as well as direct anthropogenic disturbance of natural ecological communities disable the proposed mechanism of biotic regulation (Gorshkov et al. 2000).

Broad acceptance of the genetic adaptation principle results in scientists generally ignoring empirical evidence supporting the existence of the biotic regulation of the environment. As we discuss in the following section, the adaptation concept contradicts some important empirical data that have accumulated since the concept was formulated by Darwin. 


\section{THE CLIMATE STABILITY PHENOMENA}

Any stable state is characterised not by the absolute constancy of the system's characteristics, but by the absence of irreversible changes in their values. On this basis, the most remarkable property of Earth's climate is not its variability due to the succession of glacial and inter-glacial periods, volcanic activities, drift of the continents, meteorite fall, change in solar activity etc., but the fact that, in spite of all those dramatic perturbations, the climate has remained suitable for life during the last four billion years.

Irrespective of the orbital position occupied by a planet in the solar system, the planet's surface temperature can assume almost arbitrary values depending on the values of the planetary albedo and greenhouse effect, the latter being completely determined by inherent environmental characteristics of the planet. There are two physically stable states of the Earth's climate, that of complete ice coverage with global mean surface temperature close to $T \sim-100^{\circ} \mathrm{C}$ and that of total evaporation of the Earth's hydrosphere with temperature close to $T \sim+400^{\circ} \mathrm{C}$. In both stable states life is impossible. There are strong lines of evidence that the modern climate of Earth, where the predominant part of the hydrosphere exists in the liquid state, is physically unstable with respect to spontaneous transition to either of the two life-incompatible states (Gorshkov et al. 2000; Gorshkov \& Makareiva, 2002). The documented stability of the suitable-for-life global mean surface temperature over geological timescales can thus be only explained if one allows for appearance of biotically controlled singularities in temperature-dependent behaviour of the greenhouse effect and albedo within the lifecompatible temperature interval corresponding to the liquid state of water.

In other words, the analysis of the climate stability problem allows us to conclude that, were the global biota to adapt continuously to randomly changing climatic conditions, nothing would prevent these conditions from becoming unfit for any life within geologically very short time periods. It is only the compensatory stabilising impact of the global natural biota that allows to explain the maintenance of a lifecompatible climate on Earth during the four billion years of life existence. As the natural biota of Earth are being destroyed in the course of anthropogenic activities, the climatic fluctuations start to grow, a process which may ultimately lead to an irreversible transition of the Earth's climate to one of the two life-incompatible states. In this light, it is likely that the widely-discussed anthropogenic growth of atmospheric $\mathrm{CO}_{2}$ concentration does not play any role in global climate change when considered against the background of those changes that are caused by human-induced globalscale degradation of the natural regulatory biotic potential (Makarieva 2000). In any case, without identifying the nature and the quantitative degree of stability of the modern climate it is impossible to make any forecasts about possible changes of the mean global surface temperature related to changes in the atmospheric $\mathrm{CO}_{2}$ concentrations. Given this, it is remarkable that the climate stability phenomena receive so little attention within the modern scientific community as compared, e.g., to the problem of quantifying major global sources/sinks of atmospheric carbon.

\section{SPECIES DISCRETENESS AND THE GENETIC ADAPTATION PRINCIPLE}

Another bulk of evidence against the genetic adaptation paradigm comes from the analysis of paleodata. The available paleodata point to the morphologic and, 
consequently, genetic (Jackson 1990) constancy of all species-specific characteristics during the whole period of existence of most of the species studied (Stanley 1979). In most cases, a species arises in the paleorecord as a narrow set of similar morphological forms, persists like this during the whole period of the species' existence (several million years on average) and disappears. A new related species that occupies its place in the record is separated from the old one by a considerable gap in the total set of morphological characteristics. In most cases, no transient forms that would viaduct two discrete acts of speciation are observed. The extant species also demonstrate strict discreteness. Neither intermediate forms between related species, nor processes of their formation are being observed. Hybrid zones that are sometimes considered as possible seedlings of the evolutionary process, are strongly restricted and occupy at most a few per cent of the biosphere.

The observed pattern cannot be explained by the genetic adaptation principle. Why, in spite of the assumed continuous process of adaptation to an ever-changing environment, do species retain discreteness both in space and time? Evidence for discreteness of species remains a topic largely discussed by paleobiologists, with little consideration by theoretical biologists. The revealed tempo and mode of evolutionary processes support the view that the evolution of species is not a result of gradual accumulation of relatively frequent minor modifications of the hereditary program of a species. Rather, the evolutionary process is discontinuous and is due to infrequent but radical "quantum" changes of the discrete genetic programs of species. The continuous "daily" process of mutations that supports intraspecific genetic polymorphism bears therefore no relation to the evolutionary process. Rather it can be conceived as random deviations from the stable normal hereditary program of the species. Random genetic deviations accumulate due to the mutational process and erase the genetic information of the species. The number of such deviations cannot increase infinitely, but is limited by stabilising selection. Individuals with too many genetic deviations are forced out from the population. Thus, under natural conditions the genetic program of species is prevented from decaying. This fact may explain the observed constancy of species-specific characteristics in time.

The biotic regulation principle enables observed patterns to be consistently explained from an explicit theoretical basis. Under natural conditions the maximum competitive capacity is associated with those individuals that are able to perform strictly specified complex work on stabilisation of environment conditions in tight cooperation with individuals of other species of the community. Where conditions are significantly perturbed by human activity, no environmental regulation is possible and the regulatory abilities of normal individuals no longer impart to them a competitive advantage. Hence, in distorted conditions the genetic information responsible for biotic regulation appears useless and undergoes decay, which in turn may result in an increase of intra-species genetic diversity.

For example, the majority of natural mammalian species are characterised by heterozygosity values not significantly exceeding the average 4\% (standard deviation is about 3\%) (Nevo et al. 1984; Makarieva, 2001). Meanwhile domestic animals (horses, cows, sheep) display significantly higher levels of heterozygosity, from $20 \%$ to $40 \%$ (Bowling \& Ryder 1987; Bannikova \& Zubareva 1995; Wang et al. 1990). In 
humans the average heterozygosity is four times higher than the average for the whole class of mammals. In other words, under natural conditions a huge variety of important individual properties are subject to the thorough control of natural selection, while under distorted conditions only a few of them affect the individual's competitiveness. As a result, individuals with high levels of genetic variability remain competitive in distorted environments.

\section{DISCUSSION}

We have outlined major inconsistencies that are encountered when trying to explain the available empirical evidence from different fields of the life sciences and global change science with use of the principles of (a) limiting nutrients and (b) genetic adaptation. Generally speaking, these two principles depict life as a set of objects shaped by continuous adaptation to external environmental conditions and governed by simple biochemical regularities associated with the availability of nutrients.

Both principles emerged largely as the result of scientific research into domesticated plants and animals. The concept of adaptation would not have been formulated by Charles Darwin without extensive analysis of artificial selection. Similarly, formulation of the concept of limiting nutrients owes much to agricultural and related land use practices (Liebig 1840), where it proved evident that the productivity of certain plants can be enhanced by use of chemical or organic fertilisers. However why should we expect organisms operating within natural ecological communities to behave the same as individuals in artificial conditions?

During the course of human history the biological sciences have been predominantly applied to solving the tasks of feeding humans and their medical treatment. The two conventional principles we have examined here remain perfectly valid in this context, i.e. as they apply to the functioning of separate organisms in environments dominated by human activity. However, when the same biological science is applied to global change science and applied to examining the interactions between natural biota and global environmental conditions their validity must be called into question.

An alternative view of the nature of living organisms, which emerges from analysis of the available evidence, implies that genomes of biological species contain information necessary for stabilisation of the optimal environment. Species genomes can be described as genetic programs that contain (apart from information about feeding, reproduction and other actions directly aimed at maintenance of the organism's well-being) complex information about non-random interaction with other species in the community aimed at maintenance of the optimal environment for the whole community. Such a complex internal correlation of individuals in a community can be stabilised via competitive interactions with neighbouring communities.

Within such an approach, changes in taxonomic composition of the communities due to evolutionary appearance of new species may only take place if the new species enhance (or at least do not spoil) the regulatory potential of the community. When external abiotic environment changes, there may be different taxonomic compositions of a community corresponding to the maximum possible effectiveness of environmental regulation. This opens a possibility for evolution. For example, it is 
reasonable to assume that contemporary biota are more effective in regulating modern environment with a given value of solar constant (amount of solar energy reaching the planet) than an ancient biota, which had presumably existed under conditions of significantly smaller values of solar constant.

The remarkable feature of the emerging alternative view is that it points to fundamental differences between the functioning of natural ecological communities and artificial (i.e. human influenced or managed) biosystems. If we are correct in proposing that, under natural conditions, species play unique roles in an extremely complex global environmental stabilisation mechanism, there are no grounds to expect that agricultural systems (fields, pastures, plantations etc.) that are arbitrarily composed to cater for human needs would perform the same stabilising function. On the contrary, given that these systems are generally managed to be highly productive, it is most likely that such systems act to destabilise the environment with the same or higher power as natural ecosystems stabilise it. This is confirmed by direct estimates of carbon emissions from all exploited lands.

Such a view has serious implications for elaboration of strategies to cope with current accumulation of 'anthropogenic' $\mathrm{CO}_{2}$ in the atmosphere. There is a principal difference between restoration of natural ecological communities capable of stabilising the environment and artificial replantation of the cleared areas, i.e. creation of artificial biosystems that are not only unable to support their own stability (Soberón, 1990), but, when that is done artificially by humans, represent a major destabilising factor. The ultimate goal should be thus to restore the natural regulatory function of the biota but not merely fixing in wood a certain amount of $\mathrm{CO}_{2}$, as is currently proposed (United Nations 1998).

Modern research in ecology, molecular biology and other biological disciplines is predominantly concentrated on answering the question "how living objects (cells, organisms, ecological communities) are organised" (e.g. what is the structure of biochemical reactions within a cell, internal organs in an organism, food webs in an ecosystem, etc.). Further steps in this research include modelling of the studied objects, which is associated with hopes to predict functioning of these objects under various future (environmental) conditions. However there is increasing evidence that it is not possible to develop and implement a simulation model of global change that can generate predictions sufficiently reliable to either (a) indicate that systems components, parameters and responses are sufficiently understood, or (b) form the basis of policy and management prescriptions.

Few would deny that it is impossible to adequately model any non-trivial aspect of human behaviour. It is, for example, impossible to make a model predicting future scientific discoveries made by people. In very much the same manner we argue that it is impossible to model the functioning of the natural biota, which operates with much greater information fluxes than a single Homo sapiens species. Biotic information fluxes are based on memory cells of molecular size. One bacterial cell processes an information flux compared to that flowing through a modern PC (Gorshkov et al. 2000). The biosphere contains about $10^{29}$ cells of different organisms and operates with information fluxes that are about 20 orders of magnitude (!) larger than the total information flux currently processed by the modern civilisation. It is highly 
improbable that these biotic information fluxes remain uninvolved in those processes that govern organisms' interactions with their environment. Rather it is likely that the processed information ensures the necessary reaction of organisms to environmental changes.

If simulation modelling is not a viable proposition, what roles does this leave to global change science? If the biota function along the genetic adaptation principle (based on random accumulation of new genetic variants and phenotypic properties), the biota-environment interaction is chaotic and unpredictable by definition. If, on the contrary, the biota function along the strategy of the biotic regulation of a definite optimal environment, all deviations of environmental conditions from the optimum are compensated by the natural biota up to a certain threshold determining the biota's resilience. It should be possible to quantify the time it takes biota to restore a perturbed environmental condition to the initial optimum. A second critical task for scientific investigation becomes the quantitative determination of the threshold value of destructive impact imposed on the biota by humans which is compatible with longterm environmental stability, (i.e. how much naturally functioning biota is needed to safeguard the global environment from collapse?).

We have argued that the biotic regulation principle provides a sounder basis for global change science than does the perspective resulting from jointly considering the nutrient limiting and genetic adaptation principles. If humanity were not experiencing the acute ecological problems of today, the difference between these two views on the nature of life could remain of purely academic interest. However the two paradigms lead to drastically different implications in terms of what needs to be done to address the global environmental crisis.

One interpretation based on the generally accepted paradigm is that the global biota will adapt to anthropogenic transformation as it has been adapting to spontaneous environmental changes during the four billion years of life existence. Given this, a solution to the problem of long-term environmental stability is sought in the creation of environmentally-friendly technologies that reduce the impact of modern industrial production and consumption. However this solution provides incentives for the further cultivation of the remaining natural biota and other biospheric resources, and does not recognise or value their environmental stability functions. The idea that a technological solution to the problem of global environmental security is even in principle possible, is not self-evident and demands rigorous scientific investigation. At best a technological solution is a necessary but insufficient condition.

A very different path of development compatible with long-term environmental safety follows from our proposed alternative paradigm view. It lies in the conservation and restoration of a substantial part of the Earth's biosphere in its natural unperturbed state in order to enable the stabilising potential of the natural biota of Earth with respect to the global environment will continue to function. This strategy sets a ceiling to the exploitation of biospheric resources, and places strict guidelines on the kinds of allowable economic activity and ultimately the global human population number.

Thus there is a very essential difference between the alternative strategies for human development implied by the two opposing scientific paradigms. The development path offered by the biotic regulation paradigm provides a precise 
definition of what constitutes sustainable development and the pre-requisites for a sustainable way of living. In contrast, sustainability as defined by the conventional paradigm allows complete cultivation of the biosphere and reliance on technological means of ensuring environmental stability. Irrespective of the validity of the biotic regulation paradigm, the feasibility of the technological option is entirely unproved and should not be considered as a valid option until such time as scientific investigations yield a positive and conclusive answer. Only then will humanity be free to choose between the two alternative strategies of development. Until such research is conducted, there is no free choice but only one safe strategy of development for human civilisation, namely, that of relying on the conservation of a major part of the natural biota of Earth. The technological path of development, along which modern civilisation is now spontaneously moving, is burdened with the long term risk of global ecological catastrophe following the breakdown of the unique regulatory mechanism of the natural biota.

The issues we have raised require that global biogeochemical cycles are considered jointly with phenomena such as genetics, speciation, natural selection, and community organisation. This is rarely undertaken due to the increasing specialisation of modern science. Consequently few attempts are made to envisage an integrated scientific theory of the total Earth/life system, and no one discipline takes responsibility to investigate the inconsistencies that arise when principles from different scientific fields are considered simultaneously.

All this gives good grounds for the world scientific community to urgently reevaluate the biological principles we have critiqued here that underpin global change science.

\section{ACKNOWLEDGEMENTS.}

Work of A. Makarieva is supported by grant No. 00-1596610 of the Russian Foundation for Fundamental Research.

\section{REFERENCES}

Alcamo, J. (ed.) (1994) IMAGE 2.0-integrated modelling of global climate change. Dordrecht: Kluwer Academic Publishers.

Bannikova, L.V. \& Zubareva, L.A. (1995) Genetic structure of some native and commercial breeds of cattle (Bos taurus) from Eurasia. Genetika, Vol. 31, pp. 697-708 (in Russian).

Bowling, A.T. \& Ryder, O.A. (1987) Genetic studies of blood markers in Przewalski's horse. J. Heredity, Vol. 78, pp. 75-80.

Degens, E.T., Berendt, M., Gotthardt, B. \& Reppmann, E. (1968) Metabolic fractionation of carbon isotopes in marine plankton. Deep-Sea Res. Vol. 15, pp. 11-20.

Dobzhansky, Th. (1970) Genetics of the Evolutionary Process. New York: Columbia University. Press.

Glowka, L., Burhenne-Guilmin,F. \& Synge, H. (1994) A Guide to the Convention on Biological Diversity. Gland: IUCN.

Gorshkov, V.G., Gorshkov, V.V. \& Makarieva, A.M. (2000) Biotic Regulation of the 
Environment: Key Issue of Global Change. Springer-Praxis Series in Environmental Sciences. London: Springer-Verlag.

Gorshkov V.G. \& Makarieva A.M. (1998) Impact of terrestrial and oceanic biota on the modern carbon and oxygen cycles. Ecological Chemistry, Vol. 7, pp. 129-137.

Gorshkov, V.G. \& Makarieva, A.M. (2002) Greenhouse effect dependence on atmospheric concentrations of greenhouse substances and the nature of climate stability on Earth. Atmos. Chem. Phys. Discuss., Vol. 2, pp. 289-337, see http://www.atmos-chem-phys.org/acpd.php.

Heimann, M. \& Maier-Reimer, E. (1996) On the relations between the oceanic uptake of carbon dioxide and its carbon isotopes. Global Biogeochemical Cycles, Vol. 10, pp. 89-110.

IGBP (1997) The Terrestrial Biosphere and Global Change: Implications for Natural and Managed Ecosystems. A Synthesis of GCTE and Related Research, eds. B. Walker \& W. Steffen IGBP Science N1. Stockholm: IGBP.

Jackson, J.B.C. (1990) Evolutionary significance of morphospecies: a test with cheilostome Bryozoa. Science, Vol. 248, pp. 579-583.

Keeling, R.F., Piper, S.C. \& Heimann, M. (1996) Global and hemispheric $\mathrm{CO}_{2}$-sinks deduced from changes in atmospheric $\mathrm{O}_{2}$ concentration. Nature, Vol. 381, pp. 218-221.

Liebig, J. (1840) Chemistry in its application to agriculture and physiology. London: Taylor and Walton.

Makarieva, A.M. (2001) Variance of protein heterozygosity in different species of mammals with respect to the number of loci studied. Heredity, Vol. 87, pp. 41-51.

Makarieva A.M. (2000) Biotically maintained stability of the Earth's mean global surface temperature. Petersburg Nuclear Physics Institute, Preprint No. 2384.

Nevo, E., Beiles, A. \& Ben-Shlomo, R. (1984) The evolutionary significance of genetic diversity: ecological, demographic and life history correlates. Lecture notes in biomathematics, Vol. 53, pp. 13-213.

Soberón, J.M. (1990) Deforestation and the greenhouse effect. Trends in Ecology and Evolution, Vol. 5, pp. 63-64.

Stanley, S.M. (1979) Macroevolution: pattern and process. San Francisco: Freeman.

United Nations (1998) Kyoto Protocol to the United Nations Framework Convention on Climate Change. In: COP 3 report, document FCCC/CP/1997/7/Add.1, pp. 7-30, see also http://www.unfccc.int/resource/protintr.html.

Walker, B. \& Steffen, W.S. (1997). The Terrestrial Biosphere and Global Change: Implications for Natural and Managed Ecosystems. A synthesis of GCTE and Related Research. Stockholm, Sweden: IGBP Science No. 1, 33 pp.

Wang, S., Foote, W.C. \& Bunch, T.D. (1990) Genetic variability in domesticated and wild sheep based on blood protein characters. Comp. Biochem. Physiol. B, Vol. 96, pp. 201-207. 